\title{
International
}

\section{International Membership List}

This list, as of January 2019, recognizes the countries and territories of APSA's international members.

Thank you for your continued support of the association.

\begin{tabular}{|c|c|c|c|c|c|}
\hline Argentina & 7 & Japan & 166 & Thailand & 2 \\
\hline Armenia & 1 & Kazakhstan & 1 & Tunisia & 2 \\
\hline Australia & 58 & Kuwait & 5 & Turkey & 26 \\
\hline Austria & 17 & Latvia & 1 & Ukraine & 3 \\
\hline Bangladesh & 1 & Lebanon & 9 & United Arab Emirates & 4 \\
\hline Belgium & 15 & Luxembourg & 1 & United Kingdom & 357 \\
\hline Benin & 1 & Macao & 2 & Uruguay & 6 \\
\hline Bosnia and Herzegovina & 1 & Malawi & 3 & & \\
\hline Brazil & 37 & Malaysia & 6 & Total & 2,085 \\
\hline Cameroon & 4 & Mexico & 38 & Countries & 93 \\
\hline Canada & 320 & Moldova & 1 & & \\
\hline Chile & 17 & Montenegro & 1 & & \\
\hline China & 48 & Morocco & 5 & & \\
\hline Colombia & 12 & Mozambique & 1 & & \\
\hline Côte d'Ivoire & 1 & Namibia & 1 & & \\
\hline Croatia & 1 & Nepal & 1 & & \\
\hline Cyprus & 3 & Netherlands & 47 & & \\
\hline Czech Republic & 6 & New Zealand & 8 & & \\
\hline Denmark & 49 & Niger & 1 & & \\
\hline Dem. Republic of the Congo & $0 \quad 1$ & Nigeria & 21 & & \\
\hline Dominican Republic & 5 & Norway & 39 & & \\
\hline Ecuador & 4 & Pakistan & 3 & & \\
\hline Egypt & 14 & Paraguay & 1 & & \\
\hline Ethiopia & 3 & Peru & 3 & & \\
\hline Fiji & 1 & Philippines & 2 & & \\
\hline Finland & 8 & Poland & 12 & & \\
\hline France & 34 & Portugal & 7 & & \\
\hline Georgia & 1 & Qatar & 4 & & \\
\hline Germany & 175 & Republic of Korea & 41 & & \\
\hline Ghana & 4 & Romania & 2 & & \\
\hline Greece & 2 & Russian Federation & 12 & & \\
\hline Greenland & 1 & Saudi Arabia & 1 & & \\
\hline Guam & 1 & Senegal & 1 & & \\
\hline Guatemala & 1 & Singapore & 22 & & \\
\hline Hong Kong & 25 & Slovakia & 2 & & \\
\hline Hungary & 7 & Slovenia & 3 & & \\
\hline India & 16 & South Africa & 12 & & \\
\hline Indonesia & 3 & Spain & 32 & & \\
\hline Iran & 1 & Sri Lanka & 1 & & \\
\hline Iraq & 2 & Sweden & 53 & & \\
\hline Ireland & 19 & Switzerland & 65 & & \\
\hline Israel & 39 & Taiwan & 33 & & \\
\hline Italy & 48 & Tanzania & 1 & & \\
\hline
\end{tabular}

